\title{
CLASSIFICATION OF METHODS OF THE SIGNAL SEMANTIC WAVELET TRANSFORM FOR IMAGE CONTOUR SEGMENTATION
}

\author{
Marina Polyakova ${ }^{1)}$, Victor Krylov ${ }^{2)}$ \\ 1) Applied Mathematic Department, Odessa National Polytechnic University, \\ 1, Shevchenko prospect, Odessa, 65044, Ukraine, \\ E-mail: marina_polyakova@rambler.ru \\ 2) Applied Mathematic Department, Odessa National Polytechnic University, \\ 1, Shevchenko prospect, Odessa, 65044, Ukraine
}

\begin{abstract}
In this paper the methods of signal semantic wavelet transform which underlines the edges of image in the edge detector task are analyzed and classified.
\end{abstract}

Keywords: Underlining of edges, Image contour segmentation, Wavelet transform.

Segmentation is one of tasks of the image processing, which determines the results of subsequent procedures of analysis of images: identification and recognition. As a result of analysis of literature we are selected the following basic procedures of segmentation:

- estimation of features of segmentation; statistical estimation of center of grouping of segmentation feature for the selection of homogeneous regions of image;

- underlining of bounds between the homogeneous regions of image;

- threshold or correlation-extreme processing intended for the selection of potential bounds between the regions of image;

- morphological processing of bounds of segments for the improvement of their quality.

Underlining of bounds of regions homogeneous on some feature reduces the error of determination of co-ordinates of points of bounds. Transform of segmentation feature values for underlining of bounds of image homogeneous regions is named signal semantic transform and is defined as follows [1].

Definition 1 [1]. We name as a signal semantic transform (SST) an operator, which describes functioning of the system transforming estimation of values of feature of image segmentation $g(x, y)$ and statistical estimation of center of grouping of this feature $u(x, y)$ in the image $r(x, y)$ with the underlined bounds of regions homogeneous on this feature.

Contour segmentation partitioned the image on the basis of changes of intensity of images. There are two basic groups of contour image segmentation methods [2]. The differential methods provide the low error of determination of co-ordinates of points of edges only at a low noises level. Therefore a different approach for the increase of noise stability of these methods of segmentation is applied. The correlation extreme methods provide the high noise stability.

For the decline of the error of correlation-extreme methods of image contour segmentation the edges are underlined, that diminishes an effective width of the cross-correlation function of edge and its models.

From this point of view the decision of task of segmentation includes the choice of operator of SST, providing both a low error and high noise stability. Differentiation is the simplest statement of SST which is not provide high noise stability of contour image segmentation method.

For decision of this problem of contour segmentation low pass filtering is used. The smoothing out of edges is the lack of low pass filtering and, as a result, increases the error of determination of co-ordinates of points of image edges.

At the last time at the decision of task of contour image segmentation for underlining of edges is used a wavelet transform (WT). Methods on the basis of WT provide the image scale-space representation, under which dependence of local extreme of intensity of the contrasted image is dependences both on the strength of edge of initial image and 
from the geometrical sizes of corresponding object. During realization of WT high-frequency filtering for the edges underlining and low pass filtering with different cutting frequencies for noise stability increasing is applied. The using of low pass filtering saves the problem of decline of error of determination of co-ordinates of points of edges of large size objects as for underlining of these objects edges WT of large scale is applied.

For deciding of this problem in [1] the model of the images processing by SST in distributional sense was offered. The distribution has non-integral features and can not be correctly determinate within the framework of classic theory of functions [3].

For definition of the distribution we consider the space $K$ of real functions $\varphi(x)$, each of which has continuous derivatives of all orders and finite, i.e. equals to a zero out of some limited area. Functions $\varphi(x)$ are named test functions, and $K$ - test functions space.

The distribution $f(x)$ on space $K$ is linear continuous functional from $K$ of the space of real numbers $R$, putting in accordance of test function

$\varphi(x)$ real number $(f(x), \varphi(x))=\int_{-\infty}^{\infty} f(x) \varphi(x) d x$

Distributions include functions having integral features. Set of distributions on the space $K$ is $K^{\prime}$.

Classification of the methods of signal semantic WT for the increase of noise stability and decline of error of contour images segmentation is the purpose of this work. The following tasks are decided:

- analysis and generalization of existent methods of wavelet design on the basis of the theory of distributions for the decision of the task of multiscale contour image segmentation;

- analysis and generalization of methods of forming of nonstationary wavelet transform for the decision of the task of multi-scale contour image segmentation.

We discuss the existent methods of wavelet design on the basis of the theory of distributions and their influence on the result of SST of image.

At the design of wavelets on the basis of distributions it is expedient to save spatiallyfrequency localization of WT with the use of scaling and shifting, and also to provide numeral stability of calculations with regularization of distributions [4]. The order of these operations defines the method of wavelet design on the basis of distributions theory. Instead of the regularization of the distribution for the wavelet design the multiplication on the function allowing defining values of the distribution in rupture points is used.

Let the design of wavelet on the basis of distributions consists of the following stages:
- the distribution is multiplied by a test function or function allowing to define values of the distribution in rupture points;

- a resulting function is scaled and shifted.

We assume that a mother wavelet function $\psi(x) \in K^{\prime}$ and possesses the following properties: continuous everywhere, except for the point of $x=0$; an odd; there is a point $x_{0}>0$ such, that in the vicinity $\left(-x_{0}, 0\right)$ of the point $x=0$ the function $\psi(x)$ monotonically decreases and $\psi(x) \rightarrow-\infty$ at $x \rightarrow-0$. We assume also, that an even function $\varphi(x)$ is such, that $\varphi(0)=0$. Let $\varphi(x) \in K$ or $\varphi(x)$ is bounded variation, finite, and $\lim _{x \rightarrow 0} \psi(x) \varphi(x)=0$.

Multiplying the distribution $\psi(x)$ on a test function $\varphi(x)$, we get $\widetilde{\psi}(x)=\psi(x) \varphi(x)$.

To scale and shift a resulting function:

$$
\widetilde{\psi}_{s b}(x)=\frac{1}{\sqrt{s}} \psi\left(\frac{x-b}{s}\right) \varphi\left(\frac{x-b}{s}\right),
$$

where $s>0$ is a scale parameter, $b \in R$ is a shift parameter.

WT of function $f(x)$ with a function $\widetilde{\psi}_{s b}(x)$ as a wavelet looks like:

$T f(s, b)=\int_{-\infty}^{\infty} f(x) \widetilde{\Psi}_{s b}(x) d x=\int_{-\infty}^{\infty} f(x) \frac{1}{\sqrt{s}} \psi\left(\frac{x-b}{s}\right) \varphi\left(\frac{x-b}{s}\right) d x$

The described approach to wavelets design on the basis of distributions developed in [5, 6]. In [5] is defined WT with a hyperbolical wavelet

$$
\psi(x)=\frac{1}{\pi \alpha x} G(x),
$$

where $G(x)=1(x-\varepsilon)-1(x-\gamma), \quad 1(x) \quad$ is the step function, $G(-x)=G(x), \varepsilon, \gamma$ are constants, $\alpha$ is a coefficient.

The hyperbolical wavelet is designed out on the basis of the distribution $\psi(x)=1 / x$. As a function $\varphi(x)$ allowing defining the values of the distribution in the vicinity of rupture point, is used $G(x)$ (fig. 1). On a fig. 2 the result of scaling and shifting of the wavelet $\widetilde{\psi}(x)$ from fig. $1, \mathrm{c}$ is represented.

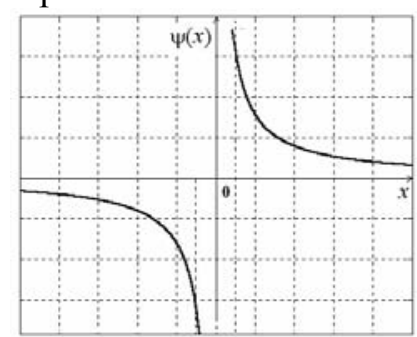

a)

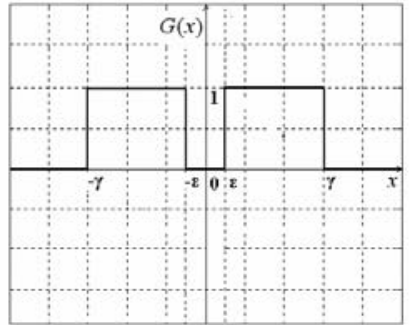

b) 


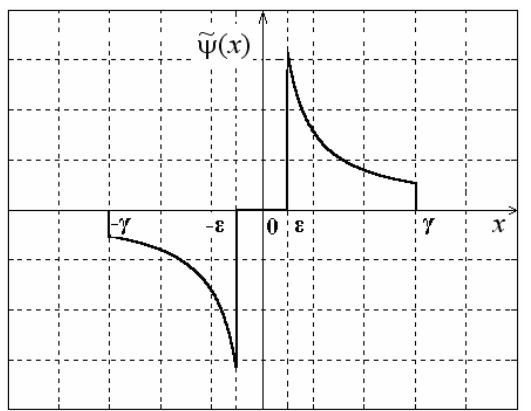

c)

Fig. 1 - The distribution $\psi(x)=1 / x$ (a), function $\varphi(x)=G(x)(\mathbf{b}) ; \widetilde{\psi}(x)=\psi(x) \varphi(x)$ (c).

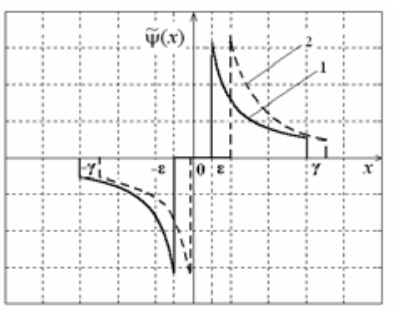

a)

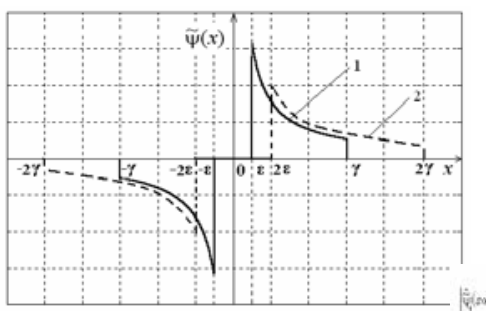

b)
Fig. 2 - Shifting with $b \geq 0$ of $\widetilde{\psi}(x)$ from a fig. 1, c (a): 1 $-b=0 ; 2-b>0$; and scaling with $s \geq 1$ (b): $1-s=1 ; 2$ $-s>1$.

The use of hyperbolical wavelet in SST for underlining of edges of image brings a more than 1 pixel edge width. Therefore methods of contour images segmentation, applying a hyperbolical wavelet for underlining of edges, irrelevant to use without the morphological processing of image edges.

To promote noise stability and to reduce the error of determination of co-ordinates of points of image edges for methods of contour segmentation in [6] it was proposed to modify a hyperbolical wavelet. This modification consists in that in place of function $G(x)=1(x-\varepsilon)-1(x-\gamma)$ allowing defining the values of the distribution in the rupture point a function $G_{1}(x)=\left\{\begin{array}{l}G(x),|x|>\varepsilon, \\ x^{2} / \varepsilon, \quad|x| \leq \varepsilon ;\end{array}\right.$ is used (fig. 3).

The graphic of the modified hyperbolical wavelet $\widetilde{\psi}_{1}(x)=(1 / x) G_{1}(x)$ on different scales is resulted on a fig. 4.

The graphics of frequency response of initial and modified hyperbolical wavelet for the different scales of WT are resulted on a fig. 5 .

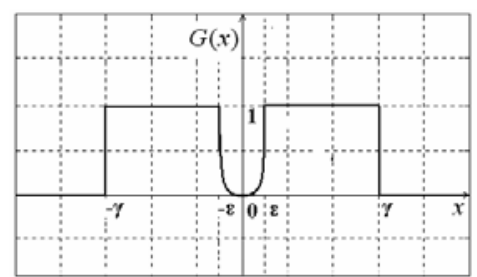

Fig. 3 - Function $G_{1}(x)$ which defines value of the distribution $\psi(x)=1 / x$ in the rupture point $x=0$.

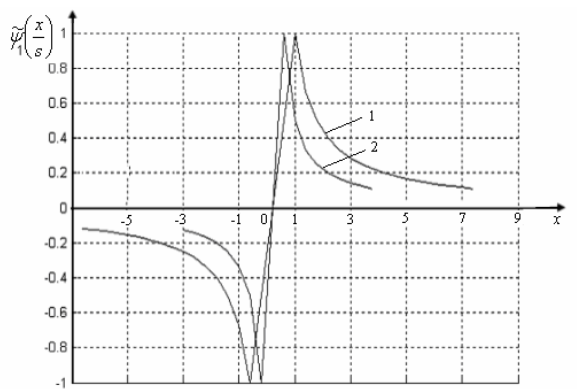

Fig. 4 - Functions $\widetilde{\psi}_{1}(x / s)$ at $s=1(1) ; 0,5(2)$.

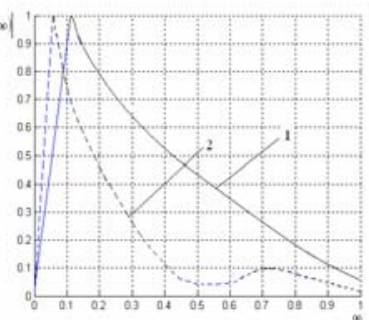

a)

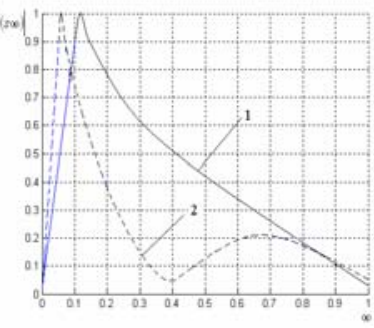

b)
Fig. 5 - Frequency response of the modified (a) and initial (b) hyperbolical wavelet for scales $s=1$ (1); 2(2).

Frequency response of the modified hyperbolical wavelet has oscillations of less amplitude and provides the best spatially-frequency localization as compared to frequency response of initial hyperbolical wavelet.

At the use of SST in space of hyperbolical wavelet in composition with the model of the multiscale images processing the noise stability of contour segmentation arises with increasing of scale of hyperbolical wavelet. However the error of determination of co-ordinates of points of edges quickly increases. The last is conditioned to those, that an analyzing hyperbolical wavelet is designed as a result of multiplication of the distribution on a function, defined of its values in the rupture points, with subsequent scaling and shifting. Change of the order of operation of the multiplication of the distribution on a function, defined of its value in the rupture point, and scaling and shifting allows decreasing the error of determination of co-ordinates of points of edges of image with increasing of scale of the wavelet.

When another method of the design of wavelet on the basis of distributions consists of the following 
stages:

- the initial distribution is scaled and shifted;

- the result is multiplied by a test function or function allowing to define values of the distribution in rupture points.

Let a mother wavelet function $\psi(x) \in K^{\prime}$ and locally integral everywhere, except for the point of $x=0$, in which $\psi(x) \rightarrow \infty$ at $x \rightarrow+0$ and $\psi(x) \rightarrow-\infty$ at $x \rightarrow-0$. We suppose also, that $\varphi(x) \in K$ and $\varphi(0)=0$. To execute scaling and shifting of the distribution $\psi(x)$ :

$$
\psi_{s b}(x)=\frac{1}{\sqrt{s}} \psi\left(\frac{x-b}{s}\right),
$$

where $s>0$ is the parameter of scale, $b \in R$ is the shift parameter.

Result of transform (3) to multiply on a test function:

$$
\widetilde{\psi}_{s b}(x)=\psi_{s b}(x) \varphi(x-b)=\frac{1}{\sqrt{s}} \psi\left(\frac{x-b}{s}\right) \varphi(x-b) .
$$

WT with $\widetilde{\psi}_{s b}(x)$ looks as:

$$
T f(s, b)=\int_{-\infty}^{\infty} f(x) \widetilde{\psi}_{s b}(x) d x=\int_{-\infty}^{\infty} f(x) \frac{1}{\sqrt{s}} \psi\left(\frac{x-b}{s}\right) \varphi(x-b) d x .
$$

This approach to wavelet design on the basis of distributions defined in [7]. The wavelet of kind (4) is called wavelet of distribution.

On a fig. 6,a the result of shifting and scaling transform of the wavelet of distribution $\widetilde{\psi}_{2}(x)=\frac{1}{x^{2}+\sqrt{x}}$ is represented.

Frequency response of the wavelet of distribution $\widetilde{\psi}_{2}(x)$ for the different scales $s$ resulted on a fig. 6 , b.

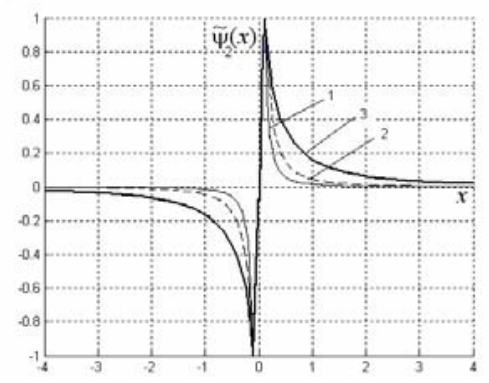

a)

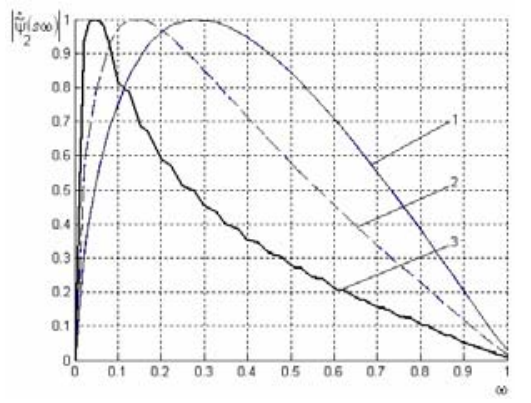

b)

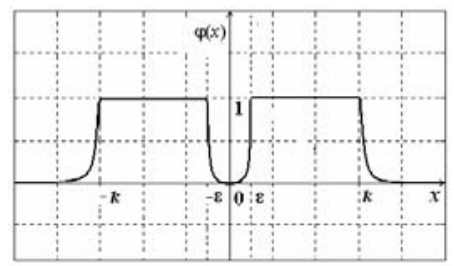

c)

Fig. 6 - Scaling of $\widetilde{\psi}_{2}(x)$ (a) with $s=1$ (a); $s=4$ (2); $s=16$ (3); frequency response of the wavelet $\widetilde{\psi}_{2}(x)$ (b) with $s=1$ (a); $s=4$ (2); $s=16$ (3); function $\varphi(x)$ which defines value of the distribution $\psi_{2}(x)$ in the rupture point $x=0$.

The pass band of the frequency response of the wavelet $\tilde{\psi}_{2}(x)$ wider than of hyperbolical wavelets $\psi(x), \psi_{1}(x)$. Therefore at the use of SST with wavelet $\widetilde{\psi}_{2}(x)$ the error of determination of coordinates of points of edges is lower than at the use of hyperbolical wavelets.

So we are analyzed of existent methods of wavelet design on the basis of the theory of distributions for the decision of the task of multiscale contour image segmentation.

The use of low pass filtering with different cutting frequency of filters allows only partly to decide the problem of decline of error of determination of co-ordinates of points of edges. The use of different wavelets on the different scales of WT is non-stationary WT and is provided the decision of this problem [8]. Families of wavelets with the single singular point also were used [9].

We discuss methods of forming of nonstationary WT and their influence on the result of the SST of the image.

In Fourier space this result in that in the place of the low pass filtering with different cutting frequencies the low pass filtering with different lifting of frequency response is applied.

For diminishing of error of determination of coordinates of points of edges at increasing of scale the lifting of the wavelet built on the basis of the homogeneous distribution, was used [10]. It is the method of modification of wavelet in a spatial domain, providing in high frequencies lifting of frequency response of wavelet.

We consider as a wavelet $\psi(x)$ the homogeneous distribution of degree $\lambda$. For example, we choose $\psi(x)=1 / x$, for which $\lambda=-1$. The further are just also in the case $\lambda \neq-1, \lambda<0$. At wavelet design on the basis of the distribution it is possible to execute scaling and shifting of the distribution, result of which to multiply on a test function. Obviously, that $\psi_{1,0}(x)=\psi(x)$, and $\varphi(x)$ we choose as in $[3,10]$.

As a result of the uniform sampling of wavelet 
$\widetilde{\psi}(x)=\psi(x) \varphi(x)$ at $x>0$ and $x<0$ we get a filter

$g_{1}=\{-1 / k, \ldots,-1 / 3,-1 / 2,-1,1,1 / 2,1 / 3, \ldots, 1 / k\}$.

If to the distribution $\psi(x)$ is applied the scaling with a scale $s>1$, then $\widetilde{\psi}_{s, 0}(x)=1 / s \psi(x) \varphi(x)$ and a corresponding filter is $g_{s}=\left\{-\frac{1}{s k}, \ldots,-\frac{1}{s \cdot 3},-\frac{1}{s \cdot 2},-\frac{1}{s}, \frac{1}{s}, \frac{1}{s \cdot 2}, \frac{1}{s \cdot 3}, \ldots, \frac{1}{s k}\right\}$.

Obviously the result of filtering of any discrete signal by the filter of $g_{s}$ is equal to the result of filtering of this discrete signal by a filter $g_{1}$, multiplied on $1 / s$. We make the lifting of filters of $g_{s}, s>1$, for the decline of error of determination of co-ordinates of points of image edges with scale increasing. It can be attained in Fourier space with a more exact approximation of differentiator $F(\omega)=-i \omega$ with the frequency response of filter $£_{s}(\omega)$.

We build the new filter of $g_{s}{ }^{\text {new }}$ with a frequency response $\xi_{s}^{\text {new }}(i \omega)=\xi_{S}(i \omega)+€_{(}(i \omega) €_{s}(i \omega), \quad$ where $\hbar(i \omega)=1$ is a frequency response of all pass filter, $€_{S}(i \omega)$ is a frequency response of high-pass filter,

$$
€_{s}(i \omega)=-q_{s} e^{-i k \omega}+q_{s} e^{-i(k+1) \omega},
$$

where $q_{s}>0, q_{s}$ is some real number. Impulse response of $g_{s}^{\text {new }}$ and its frequency response at $s=2$ resulted on a fig. 7 .

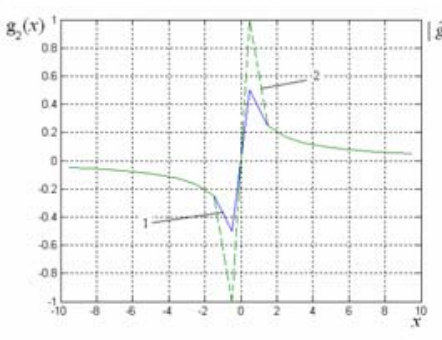

a)

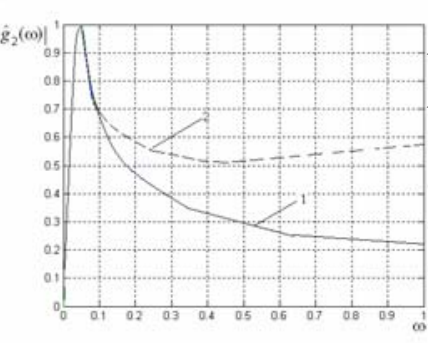

b)
Fig. 7 - Impulse response (a) and frequency response (b) at $s=2: 1$ - the filter $g_{s} ; 2$ - the lifting filter $g_{s}{ }^{\text {new }}$.

On the basis of the filter $g_{s}^{\text {new }}$ we define a wavelet

$$
\psi_{s}^{\text {new }}(x)=\psi(x / s)+\sum_{k=-\infty}^{\infty} l_{s}(k) \delta(x-k),
$$

where $l_{s}(k)$ are coefficients of filter $l_{s}, \delta(x)$ is a delta function which is the result of reverse Fourier transform of the function $\bar{E}(i \omega)=1$.

Transform with the distribution wavelet (5) allows to reduce the error of determination of coordinates of points of edges of image at the decision of task of contour segmentation due to lifting of the frequency response in high pass domain (fig. 7, b). This transform is nonstationary WT, as uses different wavelet on the different scales $s$.

At the decision of practical tasks the filters based on distributions of kind (5) were used in [11].
Contour segmentation was conducted by filters $g_{N}$, where $N$ is amount of levels of WT, $2 \leq N \leq 7$ (fig. 8).

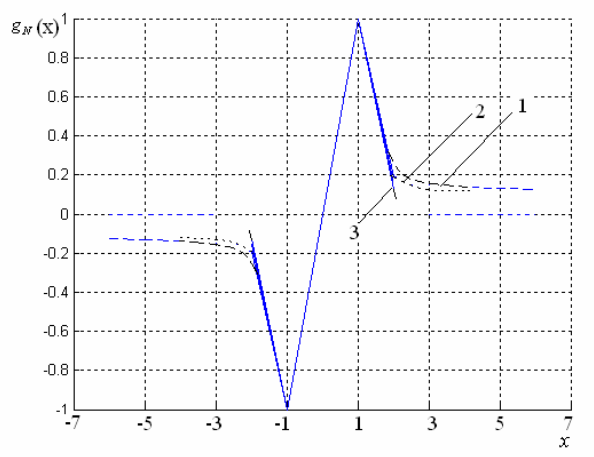

Fig. 8 - Impulse response of filter $g_{N}$ at $N=7(1) ; 5(2)$; 2(3).

We will notice that geometrically process of construction of wavelets (5), for example, in case, $\psi(x)=1 / x$ it is possible to represent also as follows. The graph of hyperbola $y=1 / x$ (fig. 9, a) will be transformed by a shift on $b_{0}>0$ : $y_{1}=1 /\left(x+b_{0}\right) \quad$ (fig. 9, b). From the graph $y_{1}=1 /\left(x+b_{0}\right)$ we leave a fragment at $x>1$, and from the graph $y=x$ fragment at $x \in[0,1]$ (fig. 9, c). We connect both graphs in the rupture point and antisymmetrical represent it on a negative semi axis $O y$ (fig. 9, d). In [11] a wavelet was built by this rule, but the graph $y_{1}=1 /\left(x+b_{0}\right)$ was additionally scaled before the selection of fragment at $x>1$.

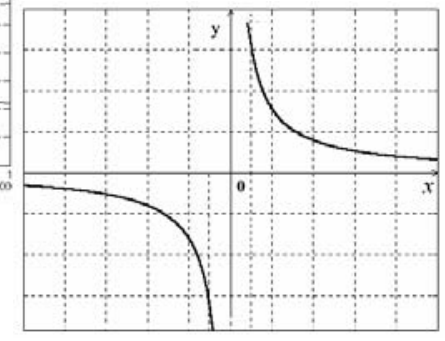

a)

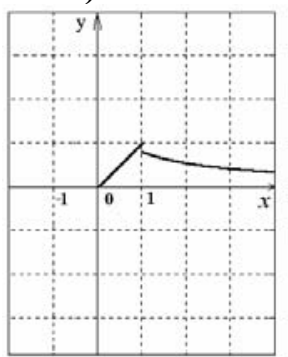

c)

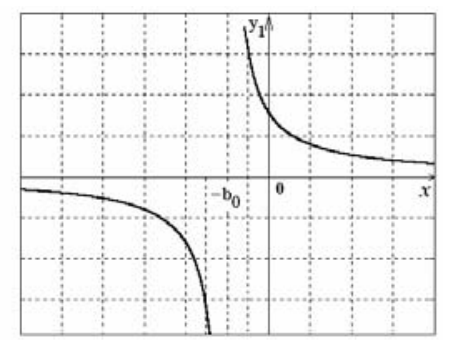

b)

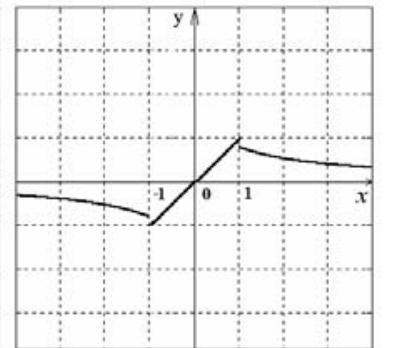

d)
Fig. 9 - Graphic of hyperbola $y=1 / x(a), y_{1}=1 /\left(x+b_{0}\right)$ (b), construction of wavelet (5) (c, d).

Examining the resulted geometrical interpretation of the wavelet of distributions (5) we notice that the last have the rupture in points $x=1, x=-1$. If to take into account only environments of point of $x=0$ kind $\left(1, x_{1}\right),\left(-x_{1}, 1\right)$, where $x_{1}>0$, it is easily to prove that 
the wavelet of distributions (5) possess by different local regularity in the point of $x=0$ depending on the scale $s$ (with the indicated limitation on environments of point of $x=0$ ). From the point of view of image contour segmentation methods using SST in space of distributions (5), ruptures of these functions in points $x=1, x=-1$ conduces to the decline of noise stability of these methods. Therefore, to provide the low error of determination of coordinates of edge points at high noise stability of method of contour segmentation, it is expedient to use SST in space of wavelet of different regularity, with a common singular point [9].

This type of WT which is named repagular (from Latin repagulum is retentive barrier) allows more exactly estimates edges of image. The change of scale of repagular WT is made not by scaling, as at ordinary wavelet transform, and by the use of functions of different regularity with one scale. It allows to save spatial localization of contour on condition of frequency localization.

Continuous repagular WT for any function $f(x) \in L_{2}(R)$, where $L_{2}(R)$ is space of functions integral with a square on the space of real numbers $R$, is defined as a convolution of function $f(x)$ with the functions $\psi(x, a)$ of different regularity, i.e.

$$
\left(T^{\text {rep }} f\right)(a, b)=\int_{-\infty}^{\infty} f(x) \psi(x-b, a) d x
$$

where $a$ is parameter characterizing regularity of function, unlike the scale of ordinary wavelet transform; $b$ is a shift determining a convolution operation.

We define functions $\psi(x, a)$ of different regularity by a unique function depending on a parameter $a$, as follows. Let $\psi(x, a) \in L_{1}(R)$, where $L_{1}(R)$ is space of functions integral on the module on the $R$, is an odd function depending on a parameter $a$. We assume that $\psi(x, a)$ has an integral feature in a point $x=0$, i.e. $|\psi(x, a)| \leq C_{a}|x|^{-a}$, $a \in(0,1), C_{a}$ is some constant and $\int_{-\infty}^{\infty} \psi(x, a) d x=0$. The example of such function is $\psi(x, a)=|x|^{-a} \operatorname{sgn}(x) w(x, a)$, where $w(x, a)=\left\{\begin{array}{l}1,|x|^{-a} \geq \varepsilon_{a}, \\ 0,|x|^{-a}<\varepsilon_{a},\end{array}\right.$ the fixed number is $\varepsilon_{a}$.

A function $\psi(x, a)$ which defines repagular WT is named a repagular wavelet (fig. 10).

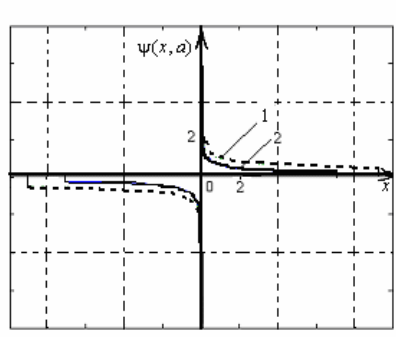

a)

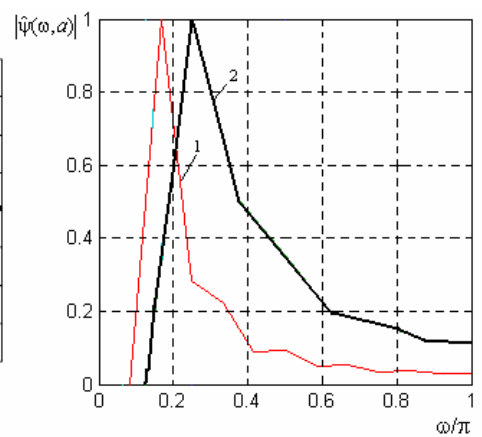

b)
Fig. 10 - Functions $\psi(x, a)$ (a) and these frequency responses (b) at $a=0,5$ (1); 0,25 (2).

Repagular wavelets are functions of different regularity, covering the range of values of functions from $\delta^{\prime}(x)$, designing differentiator to step function $\theta(x)$, as an edge model in the correlationextreme methods of contour segmentation of images. Therefore, applying these functions for image edges underlining due to the choice of parameter $a$ it is possible to get the methods of contour segmentation with different values of noise stability and error of determination of co-ordinates of points of image edges.

So we are analyzed of methods of forming of nonstationary WT for the decision of the task of multi-scale contour image segmentation.

The repagular WT also as WT based on the lifting is realized with filters with different lifting of frequency responses in high pass band. Therefore methods of signal semantic WT with realization ways are classified as follows (fig. 11).

WT used low pass filtering with different cutting frequencies provides noise stability of contrasted image for contour segmentation. The low error of determination of coordinates of points of edges is provided with WT used low pass filtering with different lifting of frequency responses in high pass band.

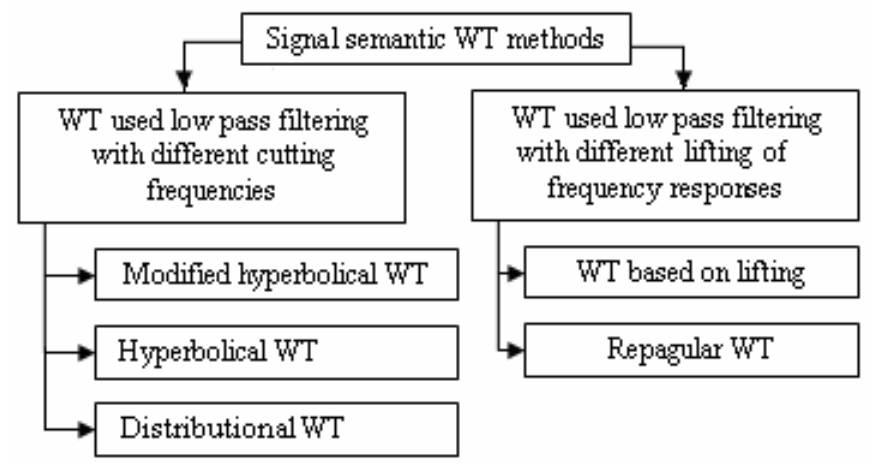

Fig. 11 - The classification of methods of signal semantic WT of images.

Thus in this paper the analysis, generalization and classification of methods of signal semantic WT for the increase of noise stability and decline of error 
of contour images segmentation is carried out.

\section{References}

[1] M.V. Polyakova, V.N. Krylov, "Hierarchical signal semantic transformation in space of distributions for the task of images segmentation" // Proc. of Odessa Politechnic University, Vol. 2 (26), pp. 161-167, 2006.

[2] V.N. Krylov, M.V. Maksimov, "Second transformers of signals of images", Odessa: Astroprint, 1997.

[3] I.M. Gelfand, G.E. Shilov, "Distributions and actions above them", vol. 1, Moscow: Fizmatgiz, 1959.

[4] V.S. Vladimirov, "Distributions in the mathematical physics", Moscow: Nauka, 1979.

[5] Y.A. Klih, S.G. Antoshchuk, and A.A. Nikolenko, "Adaptive basis functions of hyperbolical wavelet transform" // Proc. of Odessa Politechnic University, Vol. 2 (22), pp. 121-125, 2004.

[6] V.N. Krylov, M.V. Polyakova, and N.P. Volkova, "Contour segmentation in space of hyperbolical wavelet transform with the use of mathematical morphology" // Automatica. Automation. Electrical engineering complexes and systems, № 2(18), pp. 57- 63, 2006.

[7] M.V. Polyakova, V.N. Krylov, "Characterization of local regularity of functions with wavelet of distribution help" // Proc. of Odessa Politechnic University, Vol. 2 (24), pp. 192-198, 2005.

[8] Cohen A., Sere E, "Time-frequency localization with non-stationary wavelet packets" // Subband and Wavelet Transforms: Design and Application / Akansu A.N., Smith M.J.T. (eds.), Kluwer, 1996, pp. 54-81.

[9] M.V. Polyakova, V.N. Krylov, "Morphological method of contour segmentation of images on the basis of repagular wavelet transform" // Proc. of Odessa Politechnic University, Vol. 1 (25), pp. 98-103, 2006.

[10] V.N. Krylov, M.V. Polyakova, "Contour images segmentation in space of wavelet transform with the use of lifting" // Opticalelectronic informatively-power technologies, № 2(12), pp. 48-58, 2007.

[11] S.G.Antoshchuk, A.G. Derevianchenko, and E.V. Tkachenko, "The hierarchical objects analysis on images of cutting tool wear zones" // Proc. of International Conf. TCSET'2006, Lviv, Ukraine, Feb. 2006, pp. 253-255.

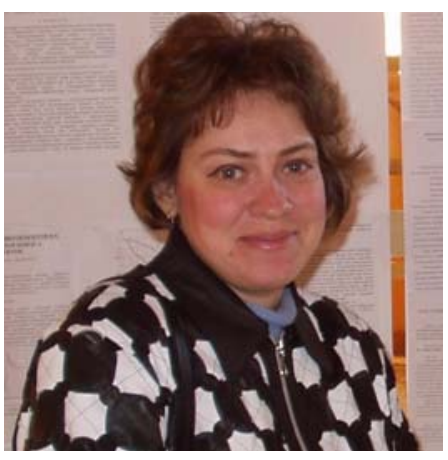

Marina Polyakova, Specialist (1994), applied mathematic, Odessa State University, candidate of technical sciences (2005), automatic control systems and progressive information technologies, Odessa National Polytechnic University, associate professor of the chair "Applied mathematic and information technologies in the business" (2006), Odessa National Polytechnic University.

Scientific interests: wavelet analysis, fractals, theory of distributions, functional analysis.

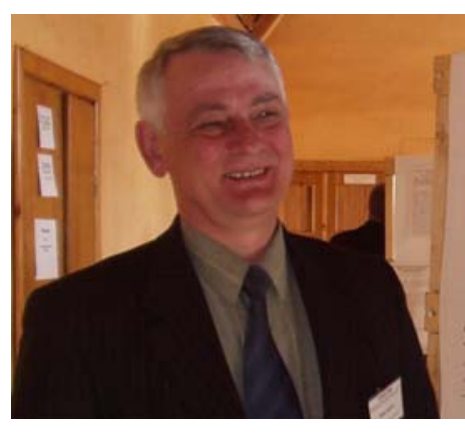

Victor Krylov, Specialist (1978), radio techniques, Odessa Polytechnic Institute, candidate of technical sciences (1986), radio technical apparatus and systems, doctor of technical sciences (2003), automatic control systems and progressive information technologies, full professor of the chair "Applied mathematic and information technologies in the business" (2005), Odessa National Polytechnic University.

Scientific interests: digital image processing, pattern recognition. 